\title{
Características de las intoxicaciones por drogas atendidas en once servicios de urgencias españoles: Análisis diferenciado por sexo
}

\section{Characteristics of drug poisonings treated in eleven Spanish emergency departments: Differentiated analysis by sex}

\author{
Miguel Galicia*, Dima Ibrahim-Achi**, Òscar Miró*, August Supervía***, Jordi \\ Puiguriguer****, María Ángeles LeciñenA*****, Ma José Venegas de L'Hotellerie******, Beatriz \\ Martín-Pérez*******, Ana Ferren*********, Guillermo Burillo-Putze*********, en representación \\ de la Red de estudio de drogas en Urgencias Hospitalarias en España (REDURHE). \\ * Área de Urgencias, Hospital Clínic, IDIBAPS, Universitat de Barcelona, Barcelona, España. \\ ** Servicio de Urgencias, Hospital Universitario de Canarias, Tenerife, España. \\ *** Servicio de Urgencias y Unidad Funcional de Toxicología, Hospital del Mar, Barcelona, España. \\ **** Servicio de Urgencias y Unidad de Toxicología Clínica, Hospital Son Espases, Palma de Mallorca, España. \\ ****** Servicio de Urgencias, Hospital Can Misses, Ibiza, España. \\ ****** Servicio de Urgencias, Hospital Rey Juan Carlos, Móstoles, España. \\ ******** Servicio de Urgencias, Hospital Río Hortega, Valladolid, España. \\ ******** Unidad de Toxicología Clínica, Hospital Clínico Universitario Lozano Blesa, Zaragoza, España. \\ ********* Departamento de Medicina Física y Farmacología, Universidad de La Laguna, Tenerife, España.
}

\section{Resumen}

Con el objetivo de identificar, con perspectiva de género, las diferencias sociodemográficas, clínicas, manejo en urgencias y gravedad de las intoxicaciones por drogas atendidas en Servicios de Urgencias Hospitalarias (SUH), se registraron todos los pacientes atendidos en 11 SUH españoles durante 24 meses (agosto 2017-julio 2019). La gravedad de la intoxicación se basó en el evento adverso combinado (intubación orotraqueal, parada cardiorrespiratoria, hospitalización en intensivos, y muerte), comparándose según el sexo. Cuando se encontraron diferencias significativas en sintomatología o gravedad, los resultados se ajustaron por características sociodemográficas y drogas consumidas. Se incluyeron 4.526 pacientes (hombres $75,5 \%$ ), con edad media de 33 años. Las drogas más frecuentes fueron cocaína $(47,8 \%)$, cánnabis $(44,4 \%)$ y anfetaminas $(25,5 \%)$. Hubo más GHB en hombres $(5,6 \%$ vs $1,9 \%, \mathrm{p}<, 001)$ y más benzodiacepinas $(8,0 \%$ vs $11,1 \%, \mathrm{p}=, 002)$ y alcohol $(57,2 \%$ vs $61,2 \%, \mathrm{p}=$ ,028) en mujeres, sin diferencias en otras de drogas. Los hombres tuvieron significativamente más bradicardia grave $(\mathrm{OR}=4,39$, IC95\% $=1,03-18,7)$, dolor torácico $(\mathrm{OR}=1,72, \mathrm{IC} 95 \%=1,27-2,35)$ e hipertensión sintomática $(\mathrm{OR}=1,56, \mathrm{IC} 95 \%=1,06-2,30)$ y menos ansiedad $(\mathrm{OR}=0,74, \mathrm{IC} 95 \%=$ $0,61-0,89)$ y vómitos $(\mathrm{OR}=0,64, \mathrm{IC} 95 \%=0,51-0,80)$. Tuvieron también más eventos adversos combinados $(3,1 \%$ vs $2,0 \%, \mathrm{p}=, 047)$ y más intubaciones $(1,9 \%$ vs $1,0 \%, p=, 044)$, pero sin diferencias significativas en el modelo ajustado $(\mathrm{OR}=1,349$, IC95\% $=0,827-2,202$ y OR $=1,371$, IC95\% $=0,700$ 2,685 , respectivamente). Fallecieron 12 pacientes $(0,3 \%)$, sin diferencias según sexo. Concluimos que existen diferencias según el sexo en las drogas que originan intoxicaciones atendidas en los SUH. Las diferencias en sintomatología cardiovascular (más en hombres) y ansiosa o digestiva (más en mujeres) no se explican por diferencias sociodemográficas o de drogas utilizadas. La gravedad de la intoxicación no se ve influida por el sexo. Palabras clave: Intoxicación; drogas; sexo; gravedad; urgencias hospitalarias.

\begin{abstract}
In order to identify the sociodemographic, clinical, emergency management and severity differences of drug poisoning treated in Emergency Departments (ED) from a gender perspective, data on patients from 11 Spanish EDs were recorded over 24 months (August 2017-July 2019). The severity of intoxication was compared by sex and was based on the combined adverse event (orotracheal intubation, cardiorespiratory arrest, intensive care hospitalization, and death). We included 4,526 patients (men $75.5 \%$ ), with a mean age of 33 years. The most frequent drugs were: cocaine $(47.8 \%)$, cannabis $(44.4 \%)$ and amphetamines $(25.5 \%)$. Men consumed more GHB $(5.6 \%$ vs. $1.9 \%, \mathrm{p}<.001)$ and less benzodiazepines $(8.0 \%$ vs. $11.1 \%, \mathrm{p}=.002)$ and alcohol $(57.2 \%$ vs. $61.2 \%, \mathrm{p}=.028)$ than women, with no differences in other types of drugs. Men presented significantly more severe bradycardia $(\mathrm{OR}=4.39,95 \% \mathrm{CI}=1.03-18.7)$, chest pain $(\mathrm{OR}=1.72,95 \% \mathrm{CI}=1.27-2.35)$ and symptomatic hypertension $(\mathrm{OR}$ $=1.56,95 \% \mathrm{CI}=1.06-2.30)$ and less anxiety $(\mathrm{OR}=0.74,95 \% \mathrm{CI}=0.61-0.89)$ and vomiting $(\mathrm{OR}=0.64$, CI95\% $=0.51-0.80)$. Men had more combined adverse events $(3.1 \%$ vs. $2.0 \%, \mathrm{p}=.047)$ and a greater intubations $(1.9 \%$ vs. $1.0 \%, \mathrm{p}=.044)$, with no significant differences in the adjusted model $(\mathrm{OR}=1.349,95 \% \mathrm{CI}=0.827-2.202$ and $\mathrm{OR}=1.371,95 \% \mathrm{CI}=0.700-2.685$, respectively). Twelve patients died $(0.3 \%)$, with no differences according to sex. Drug intoxications attended in the ED differ according to sex. GHB, benzodiazepines and alcohol are more frequently involved in men than women. Cardiovascular symptomatology is more prevalent in men, while anxiety and vomiting are more frequent in women, which cannot be explained by differences in sociodemographic characteristics or the drugs used. There were no differences in the severity of the intoxication episodes. Key words: Intoxication; drugs; sex; severity; emergency department.
\end{abstract}

Recibido: Febrero 2021; Aceptado: Julio 2021.

Enviar correspondencia a: Dr. Guillermo Burillo-Putze. Departamento de Medicina Física y Farmacología. Facultad de Ciencias de la Salud. Carretera La Cuesta-Taco, s/n, La Laguna, 38320 Tenerife, España.

E-mail: gburillo@telefonica.net 
L as encuestas realizadas en España por la Delegación del Gobierno para Plan Nacional sobre Drogas tanto a la población general, Encuesta EDADES, (2021), como a grupos específicos -Encuesta sobre uso de drogas en enseñanza secundaria, Encuesta ESTUDES, (2020) muestran una prevalencia de consumo de drogas que mantiene cifras estables o ascendentes. El 37,5\% de la población de 15 a 64 años ha probado cánnabis, el 10,9\% cocaína y el 3,1\% hipnosedantes alguna vez (cuando en 2017 estos porcentajes eran, respectivamente, del 35,2\%, 10\% y 3\%). Además, el 10,5\% había consumido cánnabis y el 2,5\% cocaína en los últimos 12 meses, siendo los hombres más consumidores de cánnabis y cocaína, y las mujeres de hipnosedantes (Delegación del Gobierno para el Plan Nacional sobre Drogas, 2021).

En el resto de Europa la situación es similar. El 29\% de los ciudadanos europeos entre 15 y 64 años (96 millones) han consumido alguna droga ilegal en su vida (cánnabis $27 \%$, anfetaminas $8 \%$, cocaína $5 \%$ ) o en el último año (cánnabis 7,6\%, anfetaminas 1,4\%, cocaína 1,3\%) (European Monitoring Centre for Drugs and Drug Addiction [EMCDDA], 2020) y, entre los estudiantes de 15-18 años, el 18\% ha consumido cánnabis en algún momento y el 5\% algún otro tipo de droga ilegal (ESPAD Group, 2020). Al igual que sucede entre la población española, el uso de drogas entre la población general es mayoritariamente masculino (EMCDDA, 2020; ESPAD Group, 2020).

Las reacciones adversas tras el consumo de drogas son una causa frecuente de consulta en los servicios de urgencias hospitalarios $(\mathrm{SUH})$, representado en algunas series hasta el 1\% del total de las asistencias (Fernández Egido, García Herrero, Romero García y Marquina Santos, 2008; Puiguriguer et al., 2013). En muchas ocasiones, Urgencias es el único contacto de los usuarios de drogas con el sistema sanitario. Por ello, los SUH son un observatorio inmejorable de las drogas que se consumen, de nuevos patrones de uso, de nuevos cuadros clínicos derivados de ello o del uso criminal de algunas de estas substancias (Burillo-Putze, López-Hernández, Expósito-Rodríguez y Dueñas-Laita, 2013; Burillo-Putze y Matos Castro, 2018; Fernández Alonso, Óscar Quintela, Ayuso Tejedor, Santiago-Sáez y González Armengol, 2019; Hegazi et al., 2017; Perelló et al., 2018).

La creación de una red de SUH que recogen asistencias secundarias al consumo de drogas permite aumentar el conocimiento sobre las características clínicas de estas intoxicaciones que acuden a los SUH españoles. El presente trabajo investiga, las diferencias entre sexos en las características de las intoxicaciones por drogas, a partir de una muestra procedente de 11 SUH españoles, tanto sociodemográficas, como del tipo de droga implicada, síntomas presentados por el paciente, manejo clínico en urgencias y gravedad del episodio.

\section{Método}

Estudio observacional descriptivo prospectivo de pacientes atendidos por sintomatología derivada del consumo de drogas de abuso en los SUH de once hospitales españoles, repartidos en seis comunidades autónomas, integrantes del grupo REDUrHE (Red de estudio de drogas en urgencias hospitalarias en España), durante un período de 24 meses (agosto de 2017-julio de 2019). Los hospitales fueron elegidos por conveniencia para formar parte de la red, en función de su trayectoria e interés previos en el estudio de pacientes intoxicados durante su atención en urgencias. El criterio de inclusión del paciente fue el de ser atendido en urgencias por sintomatología derivada del consumo de sustancias de abuso y que dicho consumo no se hubiese realizado de forma exclusiva con finalidad suicida.

Como variables sociodemográficas se recogieron: edad, sexo, modo de transporte al SUH (ambulancia o medios propios), ubicación del SUH (zona con alta incidencia de turismo lúdico -Palma de Mallorca, Tenerife, Ibiza-, gran metrópoli -Barcelona y Móstoles-, o ciudad no fundamentalmente turística -Zaragoza, Valladolid, Burgos y Salamanca-), y día (laborable o festivo) y hora (mañana: 8-16 horas; tarde: 16-24 horas; noche: 0-8 horas) de la presentación del paciente en urgencias. Como variables clínicas se recogieron: constantes vitales (frecuencia cardiaca, presión arterial, frecuencia respiratoria y temperatura) y síntomas de consulta (ansiedad, vómitos, cefalea, alucinaciones, agitación/ agresividad, psicosis, convulsiones, sintomatología cerebelosa, palpitaciones, dolor torácico, arritmias). Se definió como disminución de conciencia a una puntuación en la Escala de Coma de Glasgow de entre 9 y 14 puntos, y coma cuando ésta era $\leq 8$. Finalmente, se anotó el manejo realizado durante la estancia del paciente en urgencias (tratamiento recibido y destino al alta de urgencias).

El consumo de las drogas se determinó por la historia clínica o por analítica toxicológica mediante determinación de drogas en orina con técnicas de enzimoinmunoanálisis. Esta técnica es la disponible habitualmente en los SUH y permite determinar diversas drogas (habitualmente cannabis, cocaína, heroína, metadona, morfina, anfetaminas MDMA y benzodiacepinas) en un tiempo mínimo, con lo que se alcanza así un diagnóstico de certeza para dichas drogas. No obstante, debe tenerse en cuenta que ciertos grupos de drogas, entre los que se encuentran el gamma-hidroxibutirato (GHB) y las nuevas drogas psicoactivas, precisan de técnicas más sofisticadas y que muchos SUH basan el diagnóstico de intoxicación por ellas en la historia clínica y la sintomatología del paciente. Las drogas se agruparon siguiendo un criterio mixto (estructura química, farmacodinamia y potencial uso en medicina). Se excluyeron a los pacientes cuyo motivo de consulta fuera una intoxicación etílica pura, sin otras drogas consumidas.

Como indicador primario de gravedad se consideró cualquier combinación de los siguientes eventos adversos (EA): 
necesidad de intubación orotraqueal, parada cardiorrespiratoria, ingreso en cuidados intensivos y muerte. Cada uno de estos eventos, considerados individualmente, fueron los indicadores secundarios de gravedad.

Estos aspectos sociodemográficos, tipología de droga, sintomatología, manejo en urgencias y gravedad se compararon según el sexo. Además, respecto a la tipología de droga implicada, la prevalencia según el sexo del paciente se presenta de también forma detallada por segmentos etarios (< 20 años, 20-39 años, 40-59 años, $\geq 60$ años). Las variables cuantitativas se expresaron como media (desviación estándar (DE)) y los grupos se compararon mediante la t de Student o de la U de Mann-Whitney, y las variables cualitativas en números absolutos y porcentaje. La comparación entre grupos se llevó a cabo mediante la prueba de chi al cuadrado o el test exacto de Fisher. La magnitud de las asociaciones se calculó mediante regresión logística y se expresó como odds ratio (OR) con su intervalo de confianza del 95\% (IC95\%), primero de forma cruda y, en caso de existir significación estadística, ajustado por la edad, la coingesta de etanol y las drogas consumidas. Se consideró estadísticamente significativo un valor de $\mathrm{p}<, 05$ o si el IC95\% de la OR excluía el valor 1. Para el análisis estadístico se utilizó el paquete SPSS vs 25.0 para Windows. El estudio fue aprobado por el Comité de Ética de la Investigación del Hospital Universitario de Canarias (Tenerife), referencia 2016_71.

\section{Resultados}

Se incluyeron 4.526 pacientes (hombres: 3.418, 75,5\%; mujeres: 1.108, 24,5\%), con edad media de 33 (11) años, siendo los hombres mayores (33 vs 31 años, $\mathrm{p}<, 001$ ). La procedencia de casos según centro se detalla en la Figura 1. Las asistencias correspondieron mayoritariamente al horario nocturno $(35,3 \%)$, aunque los hombres acudieron a los SUH más en horario matinal (30,7\% vs $27,2 \%)$ y las mujeres acudieron más frecuentemente por la noche $(34,4 \%$ vs $38,2 \% ; \mathrm{p}=, 032)$. En las grandes metrópolis los hombres estaban sobrerrepresentados $(27,9 \%)$, mientras que las mujeres predominaron en los SUH de zonas de alto turismo lúdico $(55,1 \%, \mathrm{p}<, 001)$, como Baleares y Canarias.

El 39,4\% de los pacientes (1.785) consumieron dos o más drogas (media 1,52 sustancias), y 2.282 casos (58,2\%) también habían consumido alcohol etílico, hecho que resultó más frecuente en las mujeres $(61,2 \%$ vs $57,2 \%$, p =,028). Las drogas más utilizadas fueron la cocaína $(47,8 \%)$, el cánnabis $(44,4 \%)$, las anfetaminas $(25,5 \%)$, las benzodiacepinas $(8,8 \%)$, los opiáceos $(7,3 \%)$, el GHB $(4,7 \%)$ y la ketamina $(3,8 \%)$. En 254 casos $(5,6 \%)$ no se pudo identificar la sustancia implicada. Los hombres consumieron más GHB $(5,6 \%$ vs $1,9 \%, \mathrm{p}<, 001)$ y las mujeres más benzodiacepinas $(8,0 \%$ vs $11,1 \%, p=, 002)$, sin diferencias en otras drogas. El resto de las características sociodemográficas y las drogas presentes se detallan en la Tabla 1. Además, se observó que mientras que en las franjas etarias por debajo de 60 años la prevalencia de los principales tipos de drogas son muy pare-

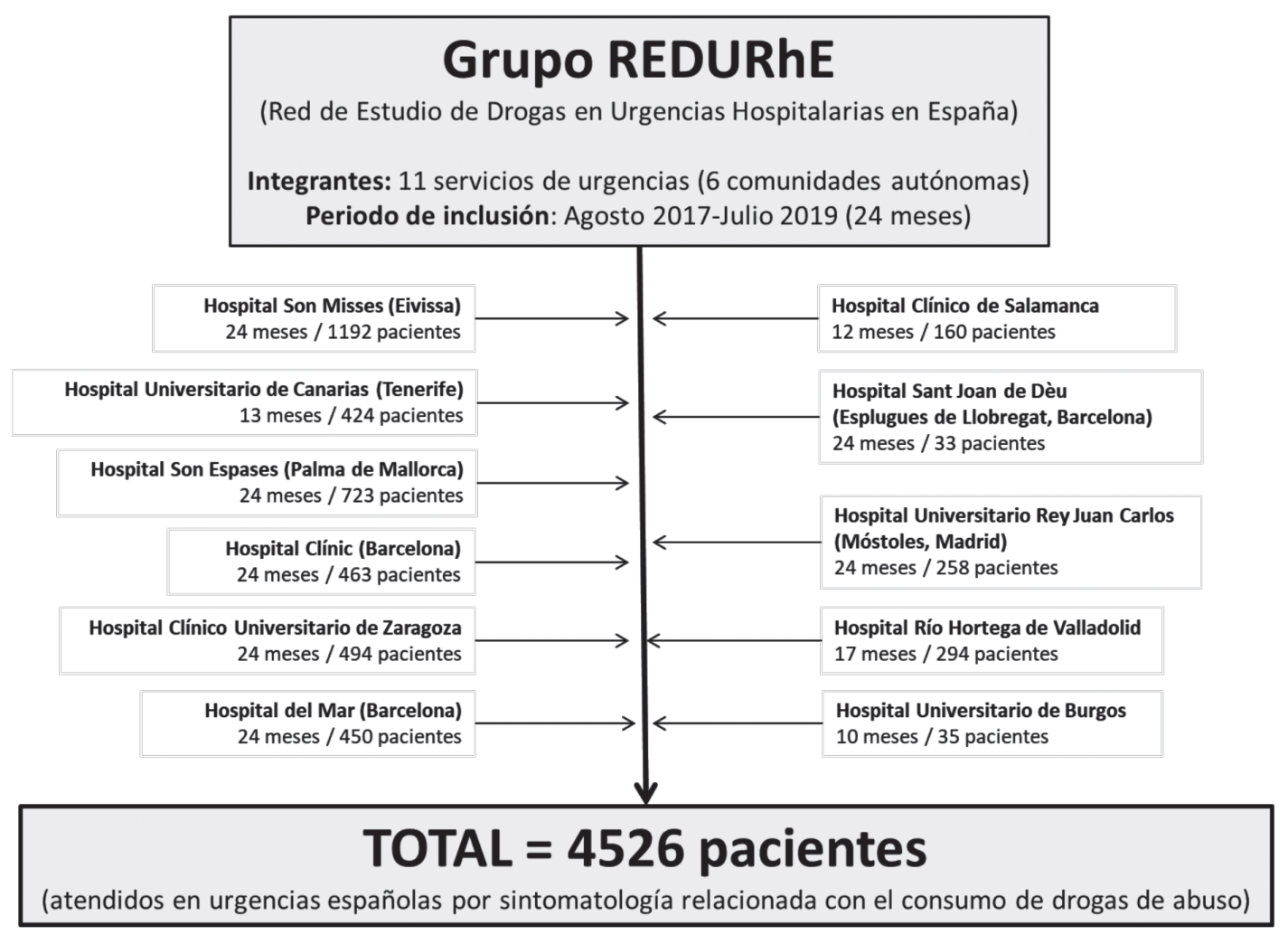

Figura 1. Diagrama de flujo de inclusión de pacientes. 
Tabla 1. Características sociodemográficas y drogas detectadas en los pacientes incluidos en el presente estudio y comparación en función del sexo del paciente.

\begin{tabular}{|c|c|c|c|c|c|}
\hline & $\begin{array}{c}\text { Total } \\
\mathrm{N}=4526 \\
\mathrm{n}(\%)\end{array}$ & $\begin{array}{c}\text { Dato ausente } \\
\text { n (\%) }\end{array}$ & $\begin{array}{c}\text { Hombres } \\
\mathrm{N}=3418 \\
n(\%)\end{array}$ & $\begin{array}{c}\text { Mujeres } \\
\mathrm{N}=1108 \\
\mathrm{n}(\%)\end{array}$ & $\boldsymbol{P}$ \\
\hline \multicolumn{6}{|l|}{ Características sociodemográficas } \\
\hline Edad (años) (media (DE)) & $32,6(11,1)$ & $5(0,1)$ & $33,1(11,3)$ & $31,1(11,3)$ & $<, 001$ \\
\hline Traídos a urgencias por el SEM & $2566(58,9)$ & $167(3,7)$ & $1952(57,1)$ & $614(55,4)$ &, 075 \\
\hline Ubicación del servicio de urgencias & & 0 & & & $<, 001$ \\
\hline En zona de alto turismo lúdico & $2339(51,7)$ & & $1728(50,6)$ & $611(55,1)$ & \\
\hline En zona de gran metrópoli & $1204(26,6)$ & & $954(27,9)$ & $250(22,6)$ & \\
\hline En zona de ciudad no turística & $983(21,7)$ & & $736(21,5)$ & $247(22,3)$ & \\
\hline Atención en urgencias en día festivo & $2218(49,0)$ & 0 & $1675(49,0)$ & $543(49,0)$ & ,999 \\
\hline Horario de atención en urgencias & & 0 & & & ,032 \\
\hline Noche $(0-8 \mathrm{~h})$ & $1598(35,3)$ & & $1175(34,4)$ & $423(38,2)$ & \\
\hline Mañana (8-16 h) & $1349(29,8)$ & & $1048(30,7)$ & $301(27,2)$ & \\
\hline Tarde $(16-24 \mathrm{~h})$ & $1579(34,9)$ & & $1195(35,0)$ & $384(34,7)$ & \\
\hline \multicolumn{6}{|l|}{ Substancias identificadas } \\
\hline Coingesta de alcohol & $2282(58,2)$ & $606(13,4)$ & $1681(57,2)$ & $601(61,2)$ &, 028 \\
\hline Coingesta de múltiples drogas & $1785(39,4)$ & 0 & $1369(40,1)$ & $416(37,5)$ & ,138 \\
\hline Número de drogas consumidas (media (DE)) & $1,52(0,73)$ & 0 & $1,53(0,73)$ & $1,48(0,71)$ &, 06 \\
\hline \multicolumn{6}{|l|}{ Drogas implicadas } \\
\hline Cocaína y derivados & $2164(47,8)$ & 0 & $1660(48,6)$ & $504(45,5)$ & ,075 \\
\hline Cannabis y derivados & $2011(44,4)$ & 0 & $1514(44,3)$ & $497(44,9)$ & ,744 \\
\hline Anfetaminas y derivados & $1156(25,5)$ & 0 & $868(25,4)$ & $288(26,0)$ & ,692 \\
\hline Benzodiacepinas & $397(8,8)$ & 0 & $274(8,0)$ & $123(11,1)$ & ,002 \\
\hline Opiáceos & $331(7,3)$ & 0 & $200(7,6)$ & $71(6,4)$ & ,183 \\
\hline Gamma-hidroxybutirato y derivados & $212(4,7)$ & 0 & $191(5,6)$ & $21(1,9)$ & $<, 001$ \\
\hline Ketamina & $174(3,8)$ & 0 & $135(3,9)$ & $39(3,5)$ &, 518 \\
\hline Fármacos psicótropos (no incluidos en otros grupos) & $64(1,4)$ & 0 & $46(1,3)$ & $18(1,6)$ & ,495 \\
\hline LSD y otras substancias alucinógenas & $31(0,7)$ & 0 & $25(0,7)$ & $6(0,5)$ &, 505 \\
\hline Nuevas drogas psicoactivas (no incluidas en otros grupos) & $14(0,3)$ & 0 & $11(0,3)$ & $3(0,3)$ & ,790 \\
\hline Otras substancias (no incluidas en otros grupos) & $57(1,3)$ & 0 & $48(1,4)$ & $9(0,8)$ & ,125 \\
\hline Substancia desconocida & $254(5,6)$ & 0 & $192(5,6)$ & $62(5,6)$ & ,978 \\
\hline
\end{tabular}

Nota. DE: desviación estándar. SEM: servicio de emergencias médicas. LSD: dietilamida de ácido lisérgico.

cidos en hombres y mujeres, esto diverge en los pacientes de 60 a más años: mientras que la cocaína y el cannabis son más frecuentes en los hombres, las benzodiacepinas y los opiáceos son más frecuentes en las mujeres (Figura 2).
Edad $<20$ años $(\mathrm{N}=\mathbf{5 1 2})$

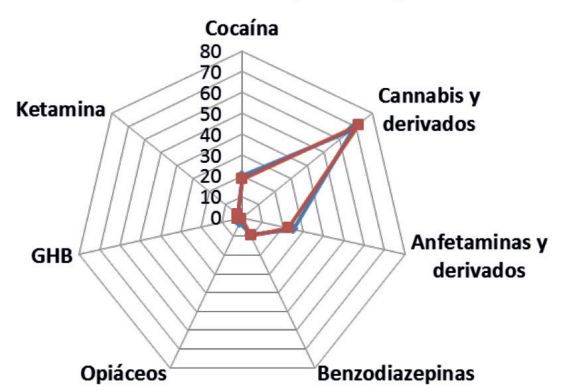

Edad 20-39 años ( $N=2.807)$

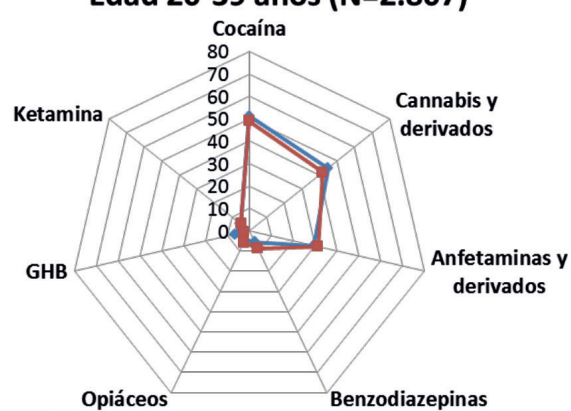

Edad $\geq 60$ años ( $N=55)$

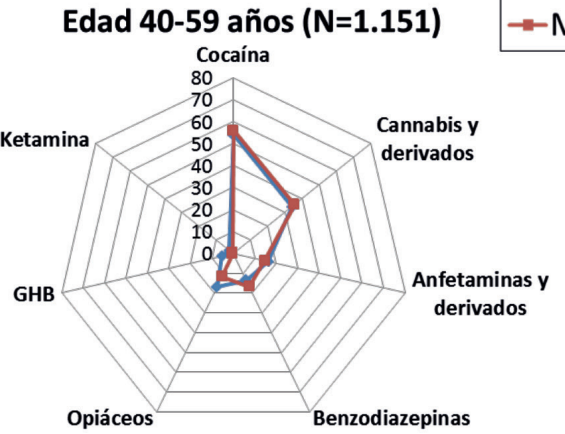

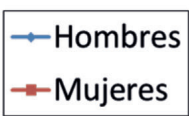

Opiáceo $62(5,6)$ 978 
Entre los síntomas, predominaron la agitación psicomotriz $(1.338$ pacientes, $29,8 \%)$ y la ansiedad (1.152 pacientes, $25,6 \%)$, aunque como puede observarse en la Tabla 2, también hubo un elevado porcentaje de pacientes con disminución de conciencia o coma (816 casos - 18,8 \% -, y 278 casos - 6,4 \% -, respectivamente). Al comparar la sintomatología entre sexos, se observó un incremento significativo en los hombres de dolor torácico, hipertensión sintomática, coma y bradicardia grave $(<50 \mathrm{lpm})$ y una mayor presencia en las mujeres de ansiedad y vómitos. La presión arterial sistólica y la temperatura a la llegada fueron mayores en los hombres (Tabla 2). En el análisis ajustado, se observó que los hombres tuvieron significativamente más dolor torácico $(\mathrm{OR}=1,72, \mathrm{IC} 95 \% \mathrm{I}=1,27-2,35)$, más hipertensión sintomática $(\mathrm{OR}=1,56, \mathrm{IC} 95 \%=1,06-2,30)$ y más bradicardia grave $(\mathrm{OR}=4,39$, IC95\% $=1,03-18,7)$, y presentaron menos ansiedad $(\mathrm{OR}=0,74, \mathrm{IC} 95 \%=0,61-0,89)$ y vómitos $(\mathrm{OR}=0,64, \mathrm{IC95} \%=0,51-0,80)$, sin diferencias en el resto de las variables analizadas (Figura 3 ).

El 76,1\% de los pacientes recibieron algún tratamiento para la intoxicación, sobre todo ansiolíticos y sedantes, que se utilizaron más en hombres $(34,6 \%$ vs 30,9\%, p = ,023). Los antídotos fueron utilizados en 351 pacientes $(7,8 \%)$, usándose más naloxona en hombres $(5,9 \%$ vs $4 \%, \mathrm{p}=, 015)$ y sin diferencias en la utilización de flumazenilo. Se determinó la presencia de tóxicos en 3.473 casos $(76,7 \%)$, ya que en el resto de casos el tóxico implicado se adjudicó a partir de los datos de la anamnesis al paciente o sus acompañantes, en presencia de un cuadro clínico (con excepción de 254 casos para los que no se pudo asignar un tipo de droga concreto, pues no se hizo historia analítica toxicológica ni hubo una historia clínica que apuntase un origen particular). No hubo diferencias en cuanto entre el porcentaje de hombres y mujeres en los que se realizó

Tabla 2. Características del episodio asistencial en urgencias los pacientes incluidos en el presente estudio y comparación en función del sexo del paciente.

\begin{tabular}{|c|c|c|c|c|c|}
\hline & $\begin{array}{c}\text { Total } \\
\mathrm{N}=4526 \\
\mathrm{n}(\%)\end{array}$ & $\begin{array}{c}\text { Dato ausente } \\
n(\%)\end{array}$ & $\begin{array}{c}\text { Hombres } \\
\mathrm{N}=3418 \\
\mathrm{n}(\%)\end{array}$ & $\begin{array}{c}\text { Mujeres } \\
\mathrm{N}=1108 \\
\mathrm{n}(\%)\end{array}$ & $\boldsymbol{P}$ \\
\hline \multicolumn{6}{|l|}{ Síntomas / Signos de consulta } \\
\hline Agitación / Conducta agresiva & $1338(29,8)$ & $33(0,7)$ & $1028(30,3)$ & $310(28,1)$ & ,149 \\
\hline Ansiedad & $1152(25,6)$ & $33(0,7)$ & $835(24,6)$ & $317(28,7)$ & ,008 \\
\hline Disminución de consciencia & $816(18,8)$ & $183(4,0)$ & $622(19,0)$ & $194(18,1)$ &, 504 \\
\hline Palpitaciones & $740(16,5)$ & $35(0,8)$ & $574(17,0)$ & $166(15,0)$ & ,133 \\
\hline Vómitos & $536(11,9)$ & $33(0,7)$ & $368(10,9)$ & $168(15,2)$ & $<, 001$ \\
\hline Síntomas psicóticos & $429(9,6)$ & $34(0,8)$ & $340(10,0)$ & $89(8,1)$ & ,051 \\
\hline Dolor torácico & $398(9,0)$ & $84(1,9)$ & $330(9,8)$ & $68(6,2)$ & $<, 001$ \\
\hline Alucinaciones & $352(7,8)$ & $36(0,8)$ & $272(8,0)$ & $80(7,2)$ & ,393 \\
\hline Convulsiones & $243(5,4)$ & $34(0,8)$ & $192(5,7)$ & $51(4,6)$ & ,179 \\
\hline Hipertensión sintomática & $242(5,0)$ & $116(2,6)$ & $197(5,9)$ & $45(4,1)$ &, 026 \\
\hline Cefalea & $181(4,0)$ & $34(0,8)$ & $130(3,8)$ & $51(4,6)$ & ,251 \\
\hline Arritmias & $123(2,8)$ & $164(3,6)$ & $91(2,8)$ & $32(3,0)$ & ,729 \\
\hline Hipotensión sintomática & $93(2,1)$ & $119(2,6)$ & $72(2,2)$ & $21(1,9)$ & ,648 \\
\hline Coma & $278(6,4)$ & $183(4,0)$ & $231(7,1)$ & $47(4,4)$ &, 002 \\
\hline Sintomatología cerebelosa & $9(0,2)$ & $51(1,1)$ & $6(0,2)$ & $3(0,3)$ &, 543 \\
\hline \multicolumn{6}{|l|}{ Constantes vitales en urgencias } \\
\hline Presión arterial sistólica (mmHg) (media (DE)) & $126(20)$ & $558(12,3)$ & $128(20)$ & $122(19)$ & $<, 001$ \\
\hline Hipotensión grave ( $(80$ mmHg) & $22(0,6)$ & & $19(0,6)$ & $3(0,3)$ & ,228 \\
\hline Hipertensión grave $(>200$ mmHg) & $10(0,3)$ & & $8(0,3)$ & $1(0,2)$ & ,730 \\
\hline Frecuencia cardiaca (lpm) (media (DE)) & $94(24)$ & $529(11,7)$ & $94(24)$ & $95(23)$ & ,283 \\
\hline Bradicardia grave ( $(50 \mathrm{lpm})$ & $40(1,0)$ & & $38(1,3)$ & $2(0,2)$ &, 004 \\
\hline Taquicardia grave (>150 lpm) & $66(1,7)$ & & $50(1,7)$ & $16(1,6)$ & ,932 \\
\hline Frecuencia respiratoria (lpm) (media (DE)) & $19(6)$ & $3684(81,4)$ & $19(6)$ & $18(5)$ & ,175 \\
\hline Taquipnea grave (>30 lpm) & $29(3,4)$ & & $24(3,6)$ & $5(2,8)$ & ,611 \\
\hline Bradicardia grave («10 lpm) & $13(1,5)$ & & $11(1,7)$ & $2(1,1)$ & ,615 \\
\hline Temperatura $(\stackrel{\circ}{C})$ (media $(D E)$ ) & $36,2(0,8)$ & $1584(35,0)$ & $36,2(0,8)$ & $36,1(0,8)$ &, 021 \\
\hline Hipotermia grave $\left(\ll 35^{\circ} \mathrm{C}\right)$ & $100(3,4)$ & & $74(3,4)$ & $26(3,5)$ & ,868 \\
\hline Hipertermia grave $\left(<40^{\circ} \mathrm{C}\right)$ & $5(0,2)$ & & $4(0,2)$ & $1(0,1)$ & 1,000 \\
\hline \multicolumn{6}{|l|}{ Manejo en urgencias } \\
\hline Administración de algún tratamiento (incluida sueroterapia) & $3412(76,1)$ & $43(1,0)$ & $2579(76,3)$ & $833(75,5)$ &, 556 \\
\hline Administración de ansiolíticos / sedantes & $1511(34,2)$ & $43(1,0)$ & $1170(34,6)$ & $341(30,9)$ & ,023 \\
\hline Administración de antídotos & $351(7,8)$ & $40(0,9)$ & $278(8,2)$ & $73(6,6)$ & ,084 \\
\hline Naloxona & $243(5,4)$ & & $199(5,9)$ & $44(4,0)$ &, 015 \\
\hline Flumaceilo & $229(5,1)$ & & $174(5,1)$ & $55(5,0)$ & ,829 \\
\hline Determinación de analítica toxicológica & $3473(76,7)$ & 0 & $2610(76,4)$ & $863(77,9)$ & ,296 \\
\hline Alta directa desde urgencias sin ingreso & $3908(87,1)$ & $33(0,7)$ & $2943(87,0)$ & $441(13,0)$ & ,704 \\
\hline Tiempo de estancias en urgencias hasta el alta (en horas) (mediana (RIC)) & $5,2(3,1-9,5)$ & $140(3,1)$ & $5,3(3,1-9,7)$ & $5,1(2,9-8,9)$ & ,124 \\
\hline
\end{tabular}

Nota. DE: desviación estándar. RIC: rango intercuartílico. 


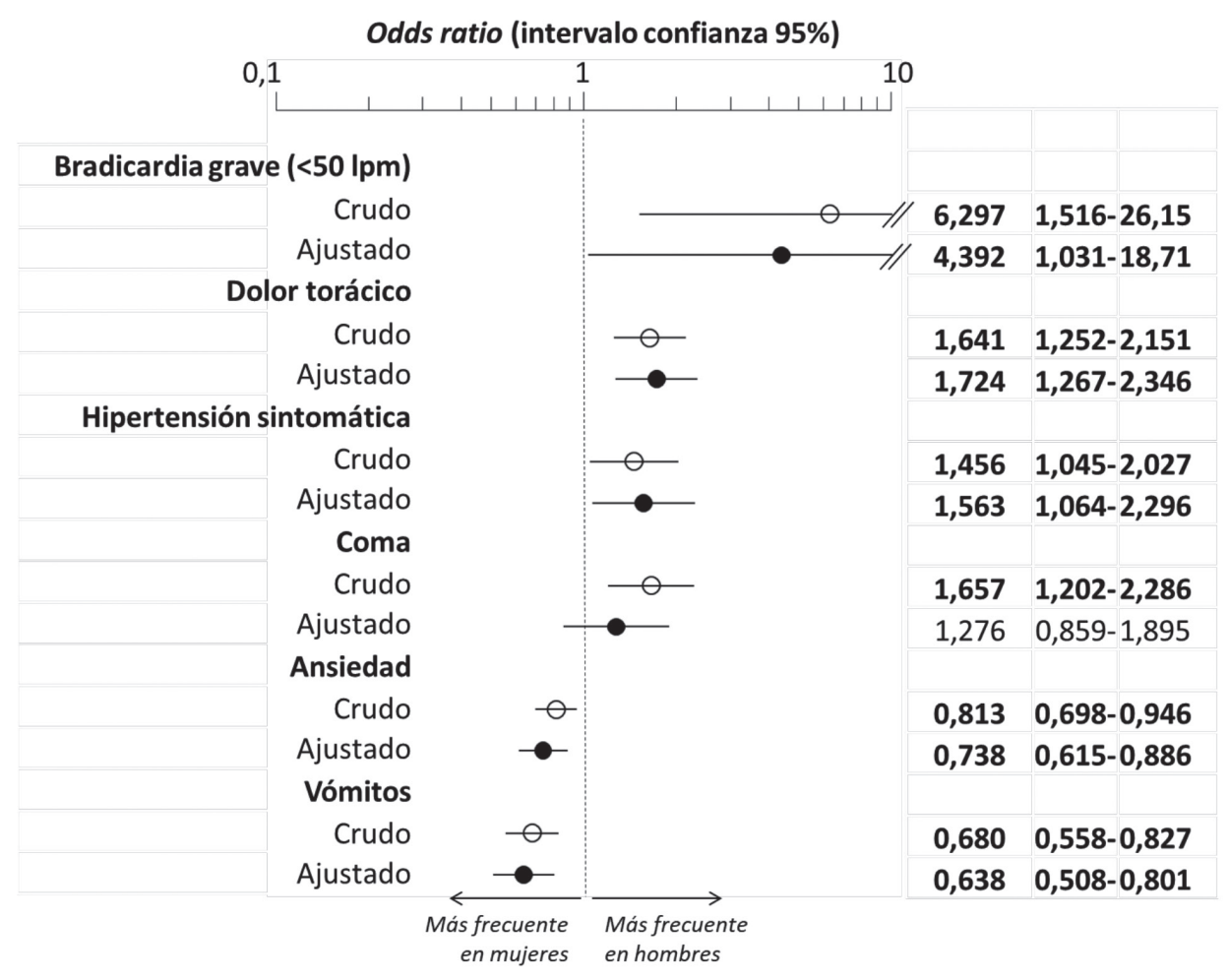

Los valores en negrita representan aquellos que alcanzan significación estadística $(p<, 05)$. Como covariables en el modelo ajustado se forzó la entrada de todos los síntomas que fueron estadísticamente significativos en el análisis crudo y además la edad, la coingesta de etanol y cada una de las drogas analizadas en el presente estudio.

Figura 3. Análisis crudo y ajustado de la sintomatología diferencial en entre hombres y mujeres que consultan a los servicios de urgencias por intoxicación por drogas de abuso.

confirmación toxicológica. Tras la asistencia en el SUH, el $87,1 \%$ de los pacientes (3.945) fueron dados de alta a domicilio, tras una estancia en el SUH de 5,4 horas de media (Tabla 2).

El EA combinado se observó en 128 pacientes (2,9\%), más frecuentemente en hombres $(3,1 \%$ vs $2,0 \%, \mathrm{p}=, 047)$ (Tabla 3). Entre los eventos adversos considerados individualmente, solo la necesidad de intubación fue diferente en función del sexo, siendo mayor en hombres (1,9\% vs $1,0 \%, p=, 044)$. En el análisis ajustado, la significación es- tadística desapareció en ambos casos ( $\mathrm{OR}=1,349$, IC95\% $=0,827-2,202$, y OR = 1,371, IC95\% = 0,700-2,685; respectivamente). Fallecieron 12 pacientes $(0,3 \%)$, sin diferencias según sexo $(0,3 \%$ vs $0,2 \%, p=, 528)$. Entre los 12 pacientes fallecidos, existió presencia de cocaína en 8 casos $(67 \%)$, derivados anfetamínicos en 3 (25\%), cannabis o derivados en $3(25 \%)$, GHB en 1 ( $8 \%$ ) y nuevas drogas psicoactivas (catinona) en $1(8 \%)$. En 6 casos $(50 \%)$, existió además coingesta de etanol.

Tabla 3. Marcadores de gravedad del episodio asistencial de pacientes atendidos por intoxicación por drogas en urgencias y comparación en función del sexo del paciente.

\begin{tabular}{|c|c|c|c|c|c|c|c|}
\hline & $\begin{array}{c}\text { Total } \\
\mathrm{N}=4526 \\
\mathrm{n}(\%)\end{array}$ & $\begin{array}{c}\text { Dato } \\
\text { ausente } \\
\mathrm{n}(\%)\end{array}$ & $\begin{array}{c}\text { Hombres } \\
\mathrm{N}=3418 \\
\mathrm{n}(\%)\end{array}$ & $\begin{array}{c}\text { Mujeres } \\
\mathrm{N}=1108 \\
\mathrm{n}(\%)\end{array}$ & $p$ & $\begin{array}{l}\text { OR cruda en hombres } \\
\text { (IC95\%) }\end{array}$ & $\begin{array}{c}\text { OR ajustada en } \\
\text { hombres } \\
\text { (IC95\%) }\end{array}$ \\
\hline \multicolumn{8}{|l|}{ Marcador primario de gravedad } \\
\hline Evento adverso combinado & $128(2,9)$ & $50(1,1)$ & $106(3,1)$ & $22(2,0)$ &, 047 & $1,595(1,002-2,537)$ & $1,349(0,827-2,202)$ \\
\hline \multicolumn{8}{|l|}{ Marcadores secundarios de gravedad } \\
\hline Parada cardiorrespiratoria inicial & $11(0,2)$ & 0 & $8(0,2)$ & $3(0,3)$ & ,829 & $0,864(0,229-3,264)$ & $0,702(0,177-2,777)$ \\
\hline Necesidad de intubación & $75(1,7)$ & $39(0,9)$ & $64(1,9)$ & $11(1,0)$ & ,044 & $1,917(1,007-3,647)$ & $1,371(0,700-2,685)$ \\
\hline Ingreso en cuidados intensivos & $90(2,0)$ & $33(0,7)$ & $73(2,2)$ & $17(1,5)$ & ,204 & $1,410(0,828-2,400)$ & $1,298(0,740-2,275)$ \\
\hline Fallecimiento & $12(0,3)$ & 0 & $10(0,3)$ & $2(0,2)$ &, 528 & $1,623(0,355-7,419)$ & $1,084(0,230-5,099)$ \\
\hline
\end{tabular}

Nota. OR: odds ratio. IC: intervalo de confianza. 


\section{Discusión}

Algunos datos epidemiológicos absolutos encontrados en el presente estudio merecen un comentario. En primer lugar, en consonancia con otras series publicadas sobre intoxicaciones por drogas atendidas en los SUH (Dines et al., 2015; Galicia et al., 2012; Miró et al., 2021), este es un fenómeno más frecuentemente observado en hombres que en mujeres (con una relación 3:1 en la serie actual). Por otro lado, también se confirma la relación del consumo de drogas con la actividad lúdica, pues resultó más frecuente en zonas turísticas, en días festivos y en horario nocturno. Finalmente, es destacable que la proporción de sexos entre los intoxicados atendidos varíe según se trate de SUH localizados en zonas más turísticas (en las que las mujeres están sobrerrepresentadas en relación a la proporción esperable) o de grandes metrópolis (en las que los que están sobrerrepresentados son los hombres). El consumo de drogas sigue pautas relacionadas con características locales, socioeconómicas o culturales, que permiten encontrar diferencias entre distintos países o regiones. Así, estudios previos han demostrado que, por ejemplo, los españoles consumen más cocaína y menos opiáceos y NPS (novel psychoactive substances) que los ciudadanos de las Islas Británicas o regiones del centro y norte de Europa (Miró et al., 2018).

Respecto a las drogas implicadas en las atenciones en los SUH, los hombres consumen más cocaína, opiáceos, GHB y ketamina, y las mujeres son más consumidoras de benzodiacepinas, si bien las diferencias solo fueron significativas para GHB (más en hombres) y benzodiacepinas (más en mujeres). En relación a este último hallazgo, debemos considerar la posibilidad de que la mayor predominancia de benzodiacepinas en mujeres se deba a un incremento de casos de uso con ideación suicida (y no exclusivamente a un uso con finalidad lúdica) o que se encuentre más fácilmente a su alcance al ser mayor la proporción de mujeres que reciben tratamiento con benzodiacepinas prescritas por un médico. Aunque los investigadores principales de cada centro fueron advertidos de que no debían incluirse los casos en los que hubiese una clara finalidad suicida en el uso de una sustancia de abuso, las características de este registro no permiten descartar esta posibilidad de forma consistente, ya que no se recogió de forma prospectiva la etiología de la intoxicación. El hecho que hayamos observado una mayor prevalencia en pacientes de 60 o más años podría sugerir este sesgo, si bien en número de pacientes correspondiente a esta franja etaria ha sido mínimo (55 del total de 4.526) y por tanto su influencia en la comparación global ha sido muy pequeña. Finalmente, es llamativo que la coingesta de etanol fuese claramente superior entre las mujeres $(61,2 \%$ vs $57,2 \%, \mathrm{p}=, 028)$. No hemos encontrado referencias similares en la literatura al respecto y, de hecho, un estudio europeo que incluyó más de 17.000 pacientes no constató esta diferencia entre sexos (Miró et al., 2021).
En relación a las manifestaciones clínicas, las más frecuentes fueron las de carácter hiperadrenérgico (agitación, agresividad, ansiedad), posiblemente debido al predominio de cocaína y anfetaminas entre las drogas consumidas (Galicia, Nogué, Sanjurjo y Miró, 2008; Galicia, Nogué, Sanjurjo y Miró, 2010; Noseda et al., 2021). Sin embargo, algunas manifestaciones clínicas particulares tuvieron diferente prevalencia en función del sexo, incluso teniendo en cuenta las diferencias sociodemográficas y el tipo de drogas consumidas por cada casa uno de los sexos. Así, hemos objetivado que los hombres son más propensos a presentar sintomatología cardiovascular: dolor torácico, hipertensión sintomática y bradicardia grave. Desconocemos si ello se relaciona con más comorbilidades cardiovasculares previas en los hombres (conocidas o silentes), pues la comorbilidad no se recogió en el estudio. Por otra parte, la ansiedad y los vómitos resultan más frecuentes en mujeres. No podemos descartar que estas diferencias de expresividad clínica puedan estar relacionadas con una susceptibilidad incrementada a ciertas manifestaciones que estuviese ligada al sexo. Así, estudios previos han mostrado que las mujeres presentan un riesgo 1,5-1,7 veces superior al de los hombres de presentar reacciones adversas en relación al uso de fármacos (Anderson, 2008; Rademaker, 2001).

Se realizó una analítica toxicológica en el $76 \%$ de los casos, sin diferencias entre sexos. Por un lado, se podría considerar que este porcentaje de analíticas toxicológicas es adecuado, ya que está demostrado que no son imprescindibles para el manejo urgente del intoxicado, pues algunos autores consideran que la analítica toxicológica debe reservarse para casos específicos (Córdoba et al., 2020). El presente estudio no muestra que exista una diferente aplicación de este criterio en función del sexo del paciente. Sin embargo, creemos que más allá de la relevancia clínica que pueda tener la determinación toxicológica, por ejemplo, revelar sustancias que el paciente no sabe que ha consumido (Liakoni et al., 2018), o existe un rol epidemiológico de dicha determinación que los SUH no debieran obviar, por su posición privilegiada a la hora de detectar nuevos patrones de consumo o la introducción de nuevas drogas en el mercado (como puede ser el caso de las NPS), para las que además deberán guardarse muestras durante el proceso asistencial para su análisis posterior mediante métodos específicos, pues su identificación escapa a las técnicas de inmunoanálisis disponibles con carácter urgente (Fernández Alonso, Quintela Jorge y Santiago Sáez, 2016; Miró y Galicia, 2019; Observatorio Español de las Drogas y las Adicciones, 2020).

En relación al tratamiento realizado en urgencias, hemos encontrado que los hombres reciben más frecuentemente fármacos ansiolíticos o sedantes, hecho que contrasta con la menor prevalencia entre ellos de sintomatología ansiosa. Esta diferencia puede tener múltiples explicaciones: la presencia de manifestaciones más pronunciadas en 
hombres o bien una mayor cantidad de cuadros de agitación, síntomas psicóticos, convulsiones o incluso hipertensión, en donde las benzodiacepinas se usan para el control de los síntomas. Los hombres también recibieron más naloxona, algo más esperable si consideramos que en números absolutos consumieron más opiáceos o GHB y que se presentaron más frecuentemente en el SUH con estados de coma (Sivilotti, 2016).

Los hombres presentaron intoxicaciones más graves, pues el riesgo de presentar un EA combinado fue un 59\% superior que en las mujeres. Entre los EA, la necesidad de intubación orotraqueal fue el único que estuvo significativamente incrementado, con aumento del riesgo del 92\% respecto a las mujeres. Esto último probablemente esté en relación con la mayor presencia de coma y de opiáceos y GHB (Galicia et al., 2008). Sin embargo, esta mayor gravedad de las intoxicaciones en hombres estaría más en relación con la mayor edad o el tipo de substancias consumidas por los hombres. Así, al ajustar por estos factores, las diferencias disminuyeron (el incremento de EA combinados disminuyó al 35\% y el de intubación orotraqueal al 37\%) y perdieron significación estadística.

Este estudio presenta limitaciones. Primera, a pesar de ser un trabajo prospectivo, existieron algunos datos que no se registraron en las historias clínicas, especialmente las constantes vitales. Siendo esto un problema generalizado en los SUH (Galicia, 2020; Miró et al., 2018; Roset Ferrer et al., 2020) debemos resaltar su importancia clínica en general y en los pacientes intoxicados en particular, donde resulta absolutamente necesario conocer y registrar tanto la temperatura (ausente en el $35 \%$ de los pacientes de esta serie, y que permite detectar casos de hipertermia) como la frecuencia respiratoria (ausente en el $81 \%$, y que permite detectar bradipnea en casos de intoxicación por GHB u opiáceos o taquipnea en casos de acidosis metabólica). Segunda, el diagnóstico de la droga se basó en la historia clínica y/o la identificación analítica, por lo que alguna sustancia pudo no ser identificada por el paciente o la analítica. Como se ha indicado previamente, algunas de las drogas, como el GHB o las nuevas sustancias psicotrópicas, no se identifican por inmunoanálisis, la técnica más usada en los SUH, y estos grupos podrían haber quedado infrarrepresentados. También pudiera ocurrir que la droga indicada por el paciente no fuese realmente la consumida, por usar otras sustancias como adulterantes de la droga. Tercera, la adjudicación de los eventos graves se realizó por el investigador de cada centro, sin monitorización externa. Los eventos considerados son muy objetivos y probablemente estén sujetos a un escaso y poco relevante sesgo interpretativo. Cuarta, a pesar de ser una serie amplia, para algunas drogas, el número de casos con alguna sintomatología o algunos EA fue escaso, por lo que pudiéramos haber incurrido en un error beta al desestimar la existencia de significación estadística en las diferencias encontradas entre hombres y mujeres. Finalmente, y pese a que los centros participantes están distribuidos por el territorio nacional, sólo hay 6 comunidades autónomas representadas, lo que limita parcialmente tener una visión global del problema en España.

En conclusión, este estudio muestra que existen diferencias según el sexo en las intoxicaciones por drogas atendidas en SUH. Existe mayor implicación de GHB en hombres y de benzodiacepinas y alcohol en mujeres. En el caso de las benzodiacepinas, esto resulta muy manifiesto en los pacientes de mayor edad. La sintomatología cardiovascular prevalece en hombres y la ansiedad y los vómitos en mujeres, y esto no podemos atribuirlo a diferencias sociodemográficas o a las drogas utilizadas por cada uno de los sexos. Los hombres presentan más EA graves, que podrían estar en relación a mayor edad y tipo y patrón de consumo de drogas, pues al ajustar por estas diferencias, la gravedad de los episodios de intoxicación no fue significativamente diferente en función del sexo.

\section{Financiación}

Trabajo financiado por la Delegación del Gobierno para el Plan Nacional sobre Drogas, Secretaría de Estado de Sanidad, Ministerio de Sanidad, ref 2016/072.

\section{Reconocimientos}

Investigadores y centros de la Red de Estudio de Drogas en Urgencias Hospitalarias en España (REDUrHE): Hospital Universitario de Canarias, Tenerife: Guillermo Burillo-Putze, Dima Ibrahim-Achi, Guillermo Castro Gainett, María Ángeles López, Aceysle González, Sebastián Matos; Hospital Clìnic, Barcelona: Miguel Galicia, Òscar Miró, Emilio Salgado, Montserrat Amigó, Santiago Nogué; Hospital del Mar, Barcelona: August Supervía, Ma Dolors Aranda, Patricia Gallardo; Hospital Son Espases, Palma de Mallorca: Jordi Puiguriguer, Christopher Yates, Juan Ortega Pérez; Hospital Can Misses, Ibiza: María Ángeles Leciñena; Hospital Sant Joan de Déu, Barcelona: Lidia Martínez Sánchez; Hospital Rey Juan Carlos, Móstoles: Ma José Venegas de L'Hotellerie, Belén Rodríguez Miranda, Santiago Kassem García, Esther Rodríguez Andrada, Rosa Wolgeschaffen Torres; Hospital Clínico de Salamanca: Ángel Bajo, Vega Riesgo Cuadrado; Hospital Río Hortega, Valladolid: Beatriz Martín-Pérez, Antonio Dueñas-Laita; Hospital Clínico Universitario Lozano Blesa, Zaragoza: Ana Ferrer; Hospital Universitario de Burgos: Francisco Callado; Hospital Universitari de Girona Doctor Josep Trueta, Girona: Cristina Ramió, María Ángels Gispert, Laia Ferrer Caballé; Hospital de Navarra, Pamplona: Miguel Ángel Pinillos; Hospital Clínico Universitario de Valencia: Benjamín Climent, Fernando Alonso; Hospital Insular de El Hierro: $\mathrm{M}^{\mathrm{a}}$ Luisa Iglesias; Complejo Hospitalario de Toledo: Natividad 
Laín; Hospital General Universitario de Alicante: Rogelio Pastor.

\section{Conflicto de intereses}

Ningún autor del presente estudio ha manifestado tener conflicto de intereses alguno.

\section{Referencias}

Anderson, G. D. (2008). Gender differences in pharmacological response. International Review of Neurobiology, 83, 1-10. doi:10.1016/S0074-7742(08)00001-9.

Burillo-Putze, G., López-Hernández, A., Expósito-Rodríguez, M. y Dueñas-Laita, A. (2013). Sumisión química, oportunista o premeditada. Medicina Clínica, 140, 139. doi:10.1016/j.medcli.2012.05.035.

Burillo-Putze, G. y Matos Castro, S. (2018). Los servicios de urgencias como atalaya de los patrones de uso de drogas y sus consecuencias clínicas. Emergencias, 30, 377-379.

Córdoba, F., Iglesias Lepine, M. L., García Gibert, L., Gispert, M. À., Moreno, A. y Supervía, A. (2020). Grado de conocimiento de la detección de drogas en orina entre médicos que atienden a pacientes intoxicados. Emergencias, 32, 451-452.

Delegación del Gobierno para el Plan Nacional sobre Drogas. (2021). Encuesta sobre alcohol y otras drogas en España, EDADES 2019/20. Madrid: Ministerio de Sanidad. Recuperado de https://pnsd.sanidad.gob.es/profesionales/ sistemasInformacion/sistemaInformacion/pdf/2019 20_Informe_EDADES.pdf.

Delegación del Gobierno para el Plan Nacional sobre Drogas. (2020). Encuesta sobre Uso de Drogas en Enseñanzas Secundarias en España (ESTUDES) 1994-2018. Madrid: Ministerio de Sanidad. Recuperado de https://pnsd.sanidad.gob.es/profesionales/sistemasInformacion/sistemaInformacion/pdf/ESTUDES_2018-19_Informe.pdf.

Dines, A. M., Wood, D. M., Yates, C., Heyerdahl, F., Hovda, K. E., Giraudon, I.,... Euro-DEN Research Group (2015). Acute recreational drug and new psychoactive substance toxicity in Europe: 12 months data collection from the European Drug Emergencies Network (Euro-DEN). Clinical Toxicology, 53, 893-900. doi:10.3109/1 5563650.2015 .1088157$.

ESPAD Group (2020). ESPAD Report 2019: Results from the European school survey project on alcohol and other drugs, Luxembourg: EMCDDA joint publications. Publications office of the European Union.

European Monitoring Centre for Drugs and Drug Addiction [EMCDDA]. (2020). European drug report 2020: Trends and developments. Luxembourg: Publications office of the European Union.

Fernández Alonso, C., Óscar Quintela, J., Ayuso Tejedor, S., Santiago-Sáez, A. E. y González Armengol, J. J. (2019).
Intoxicación aguda por nuevas drogas de abuso en probables casos de sumisión química oportunista o mixta y chemsex en pacientes con VIH atendidos en urgencias. Emergencias, 31, 289-290.

Fernández Alonso, C., Quintela Jorge, O. y Santiago Sáez, A. S. (2016). Identificación de la droga de abuso flakka ( $\alpha$-PVP) como sospecha de sumisión química no probada en urgencias de un hospital terciario. Medicina Clínica, 147, 227-228. doi:10.1016/j.medcli.2016.03.028.

Fernández Egido, C., García Herrero, G., Romero García, R. y Marquina Santos, A. J. (2008). Intoxicaciones agudas en las urgencias extrahospitalarias. Emergencias, 20, 328-331.

Galicia, M. (2020). Efectos adversos agudos en usuarios de drogas sintéticas: Una aproximación a la magnitud del problema en España. Emergencias, 32, 7-8.

Galicia, M., Nogué, S., Casañas, X., Iglesias, M., Puiguriguer, J., Supervía, A.,... Miró, Ò. (2012). Multicenter assessment of the revisit risk for a further drug-related problem in the emergency department in cocaine users (MARRIED-cocaine study). Clinical Toxicology, 50, 176182. doi:10.3109/15563650.2012.658917.

Galicia, M., Nogué, S., Sanjurjo, E. y Miró, Ò. (2008). Evolución de las consultas urgentes relacionadas con el consumo de cocaína durante el periodo 2002-2007. Emergencias, 20, 385-390.

Galicia, M., Nogué, S., Sanjurjo, E. y Miró, Ò. (2010). Consultas urgentes derivadas del consumo de éxtasis (3,4-metilenodioximetanfetamina) y derivados anfetamínicos: Perfil epidemiológico, clínico y evolutivo. Revista Clínica Española, 210, 371-378. doi:10.1016/j. rce.2010.01.013.

Galicia, M., Nogué, S., To-Figueras, J., Echarte, J. L., Iglesias, M. L. y Miró, Ò. (2008). Intoxicaciones por éxtasis líquido atendidas en servicios de urgencias hospitalarios de la ciudad de Barcelona durante 2 años. Medicina Clínica, 130, 254-258. doi:10.1157/13116549.

Hegazi, A., Lee, M. J., Whittaker, W., Green, S., Simms, R., Cutts, R.,... Pakianathan, M. R. (2017). Chemsex and the city: Sexualised substance use in gay bisexual and other men who have sex with men attending sexual health clinics. International Journal of STD and AIDS, 28, 362-366. doi:10.1177/0956462416651229.

Liakoni, E., Yates, C., Dines, A. M., Dargan, P. I., Heyerdahl, F., Hovda, K. E.,... Euro-DEN Plus Research Group (2018). Acute recreational drug toxicity: Comparison of self-reports and results of immunoassay and additional analytical methods in a multicenter European case series. Medicine, 97, e9784. doi:10.1097/ MD.0000000000009784.

Miró, Ò. y Galicia, M. (2019). Sobre las fuentes de conocimiento del consumo de drogas y sus consecuencias. Revista Española de Salud Pública, 90, 1-3. 
Miró, Ò., Waring, W. S., Dargan, P. I., Wood, D. M., Dines, A. M., Yates, C.,... Euro-DEN Plus Research Group (2021). Variation of drugs involved in acute drug toxicity presentations based on age and sex: An epidemiological approach based on European emergency departments. Clinical Toxicology. Avance de publicación on-line. doi:10.1080/15563650.2021.1884693.

Miró, Ò., Yates, C., Dines, A. M., Wood, D. M., Dargan, P. I., Galán, I.,... Galicia, M. (2018). Comparación de las urgencias atendidas por drogas de abuso en dos servicios de urgencias españoles con las atendidas en tres áreas europeas distintas. Emergencias, 30, 385-394.

Noseda, R., Schmid, Y., Scholz, I., Liakoni, E., Liechti, M. E., Dargan, P. I.,... Euro-DEN Research Group (2021). MDMA-related presentations to the emergency departments of the European Drug Emergencies Network plus (Euro-DEN Plus) over the four-year period 2014-2017. Clinical Toxicology, 59, 131-137. doi:10.1080/15563650. 2020.1784914.

Observatorio Español de las Drogas y las Adicciones. (2020). Informe 2020. Alcohol, tabaco y drogas ilegales en España. Sistemas de alerta temprana. Esquema del sistema español de alerta temprana. Madrid: Ministerio de Sanidad. Recuperado de https://pnsd.sanidad.gob.es/profesionales/sistemasAlerta/pdf/2020_Informe_Alertas.pdf.

Perelló, R., Aused, M., Saubí, N., Quirós, C., Blanco, J. L., Martínez-Rebollar, M.,... Nogué, S. (2018). Intoxicación aguda por drogas de abuso en el paciente VIH: Papel del chemsex. Emergencias, 30, 405-407.

Puiguriguer, J., Nogué, S., Echarte, J. L., Ferrer, A., Dueñas, A., García, L.,... Burillo-Putze, G. (2013). Mortalidad hospitalaria por intoxicación aguda en España (EXITOX 2012). Emergencias, 25, 467-471.

Rademaker, M. (2001). Do women have more adverse drug reactions? American Journal of Clinical Dermatology, 2, 349-351. doi:10.2165/00128071-200102060-00001.

Roset Ferrer, C., Gomila Muñiz, I., Elorza Guerrero, M. Á., Puiguriguer Ferrando, J., Leciñena Esteban, M. Á., Tuero León, G.,... Barceló Martín, B. (2020). Intoxicaciones por anfetamina y metanfetamina atendidas en los servicios de urgencias: Características clínicas y utilidad de la confirmación analítica. Emergencias, 32, 26-32.

Sivilotti, M. L. A. (2016). Flumazenil, naloxone and the "coma cocktail." British Journal of Clinical Pharmacology, 81, 428-436. doi:10.1111/bcp.12731. 